\title{
Freezing of water drops on a cold surface
}

\author{
M. E. R. Walford, D. M. Hargreaves, S. Stuart-Smith \\ H. H. Wills Physics Laboratory, University of Bristol, Bristol BS8 1TL, England \\ M. Lowson \\ Department of Aeronautical Engineering, University of Bristol, Bristol BS8 1TL, England
}

\begin{abstract}
A drop of water falling gently on to a cold copper surface freezes to it, forming a curious minaret-shaped pellet of ice. The shear tractive force necessary then to remove the ice pellet depends on the initial temperature of the metal: it is a maximum at $-22^{\circ} \mathrm{C}$ and falls to zero at $-62^{\circ} \mathrm{C}$. At impact velocities greater than approximately $0.8 \mathrm{~m} \mathrm{~s}^{-1}$, depending on the metal temperature, the droplets of water freeze to form irregular pancakes or discs of ice which adhere to the metal weakly, if at all.
\end{abstract}

\section{INTRODUCTION}

The freezing of water drops on to cold metallic surfaces and their subsequent strength of adhesion are matters of practical importance in, for example, aeronautical engineering. Bari and Hallett (1974), in their study of the rapid freezing of water drops, concentrated on the velocity regime from 0.5 to $6 \mathrm{~ms} \mathrm{~s}^{-1}$, in which regime splattered discs formed, with secondary droplets appearing at the highest speeds. For surface temperatures above $-40^{\circ} \mathrm{C}$, the discs adhered so firmly that they shattered on removal. Between $-40^{\circ}$ and $-60^{\circ} \mathrm{C}$, they adhered but could be prised off with care. They did not adhere at temperatures less than $-60^{\circ} \mathrm{C}$.

In the present paper we describe experiments in which a drop lands upon a cold metal surface at a low speed, less than $0.5 \mathrm{~m} \mathrm{~s}^{-1}$, and so forms a cylindrically symmetrical ice pellet of reproducible shape. We observe the freezing and then measure the tangential force required to remove it. The experiments give measurements of the adhesion of ice which are sufficiently reproducible to be of value.

\section{EXPERIMENTAL RESULTS}

Preliminary qualitative observations showed that freezing occurred in a similar manner over a range of drop sizes and irrespective of whether the drop fell on a copper, aluminium, brass, or aluminium alloy surface.

For quantitative measurements, we prepared apparatus, illustrated in Figure 1, which permitted visual and photographic observation of the freezing process and also permitted reproducible measurements of the shear force necessary for its removal. The metal working surface was the upper end of a rod of diameter $30 \mathrm{~mm}$. To avoid significant effects from draughts, the upper surface of the rod was set flush with the surface of a horizontal sheet of plywood $30 \mathrm{~cm}$ square and the apparatus stood in a corner of the laboratory well away from perceptible draughts. The rod's length was $300 \mathrm{~mm}$ and the lower end was immersed in a flask of liquid nitrogen. A heating coil wound around the rod just below the working surface controlled the surface temperature, $T$, within the range $-20^{\circ}$ to $-70^{\circ} \mathrm{C}$. $T$ was measured accurate to $\pm 1^{\circ} \mathrm{C}$ using a calibrated thermocouple junction, the other junction being referenced to an ice-point cell. Experiment showed that the best temperature control was obtained with a copper rod probably because of its high thermal conductivity. We therefore carried out our subsequent measurements using copper.

Measurements of adhesive forces are well known to be sensitive to the manner in which the surfaces are prepared and to their cleanliness. The most obvious problem encountered in the present experiments was that frosting occurs rapidly on a cold metal surface exposed to the atmosphere. A dry, inert atmosphere could not easily be provided, of course, because of the necessity of introducing the water drop. However, the following preparation was found by experiment to give satisfactorily reproducible measurements. The working surface was initially machined smooth using a lathe and was polished each day with a clean dry cloth to remove any dust and grease; otherwise, it was not handled. No attempt was made to avoid the formation of surface oxide layers, etc., by chemical reaction with the atmosphere. Just prior to each experiment, the heavy frost accumulated on the cold surface was scraped off as far as possible, using a glass slide. This, having low thermal diffusivity, caused no observable disturbance of the thermal state. It did, however, leave striations in the residual frosting. In order to keep frosting levels consistent between experiments, the surface was breathed upon just prior to each experiment; this 


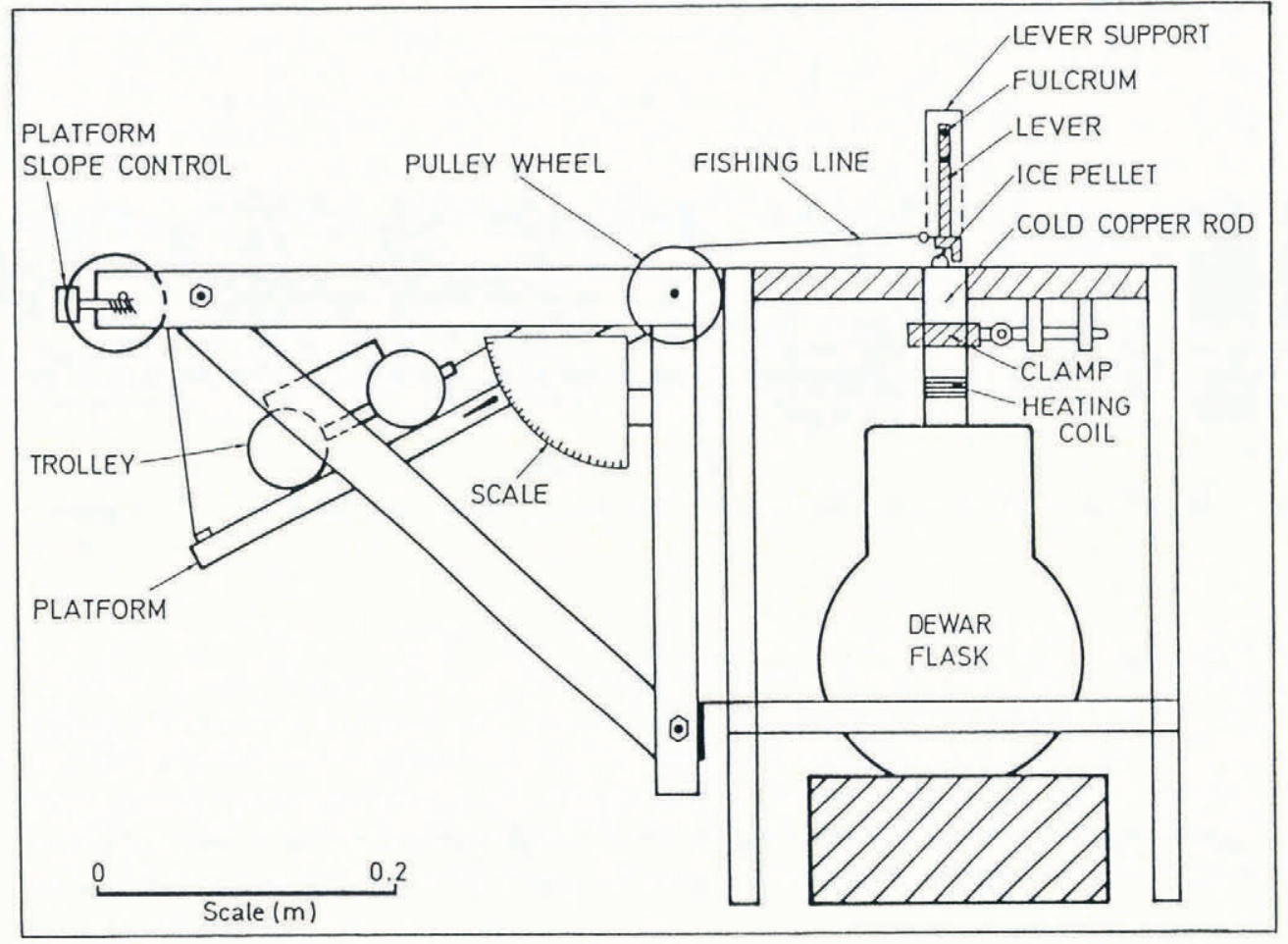

Fig. 1. The experimental apparatus for freezing the water drops and then measuring the shear force necessary for their detatchment.

produced a fine, even, frost layer.

A screw-driven syringe was used to deliver an individual drop of tap water at laboratory temperature, in a controlled manner; the drop sizes were found to vary randomly from 40 to $65 \mathrm{~mm}^{-3}$ in volume. The drop, being relatively warm, lands on the cold surface, melting and engulfing the frost underneath it, and spreading out to form a dome of water. The diameter of the contact circle is smaller the colder is the metal surface, suggesting perhaps that it is determined by competition between the processes of inertial spreading and freezing at the interface, with surface tension playing a minor role. The contact angle, $\gamma$, in the water varied inversely with the size of the contact area, between approximately $50^{\circ}$ and $130^{\circ}$.

The droplet freezes from the base upwards, taking 10 or $20 \mathrm{~s}$ to produce an opaque ice pellet with a curiously pointed pinnacle on top, like that of a minaret (Fig. 2). We measured, with a stopwatch, the time of freezing from the instant the droplet landed on the surface to the instant when freezing was completed. During freezing, the profile of the freezing drop showed no perceptible break in slope at the freezing front. One could halt the freezing process about half-way through, delicately removing the unfrozen water with a syringe or blotting paper in order to examine the ice-water interface. It was found to be rather flat, dished slightly upwards around the edge, such that the ice-water interface was approximately perpendicular to the external surface of the freezing drop. (This probably implies that at this stage the freezing process is dominated by the conduction of latent heat of fusion, down through the ice to the cold metal surface underneath.) If the freezing is allowed to proceed without interruption to completion, the final formation of the sharp pinnacle occurs with remarkable rapidity. The profile of the pinnacle varies with the conditions. Sometimes it is conical with semi-angle between $68^{\circ}$ and $72^{\circ}$. But under different conditions it has a concave profile and is drawn up into a fine point. For example, a $2 \mathrm{~mm}$ diameter drop freezing on to a surface at $-6^{\circ} \mathrm{C}$ produced a semi-angle of only $25^{\circ}$. Feathery crystals of frost accumulate on the tip. Probably, the pinnacle occurs because, during the final stage of freezing, the dominant mechanism of heat transport is no longer conduction down through the ice from the freezing water surface. Instead, heat flows convectively to the surrounding atmosphere, which is already locally chilled by the nearby metal surface. We could not observe the ice-water interface during the rapid final phase of freezing but we suspect it forms an almost closed shell. Expansion of the enclosed water on freezing would account for the sharp pinnacle.

After freezing, a photograph was taken of the ice pellet from which its height, $h$, was determined. The profile of

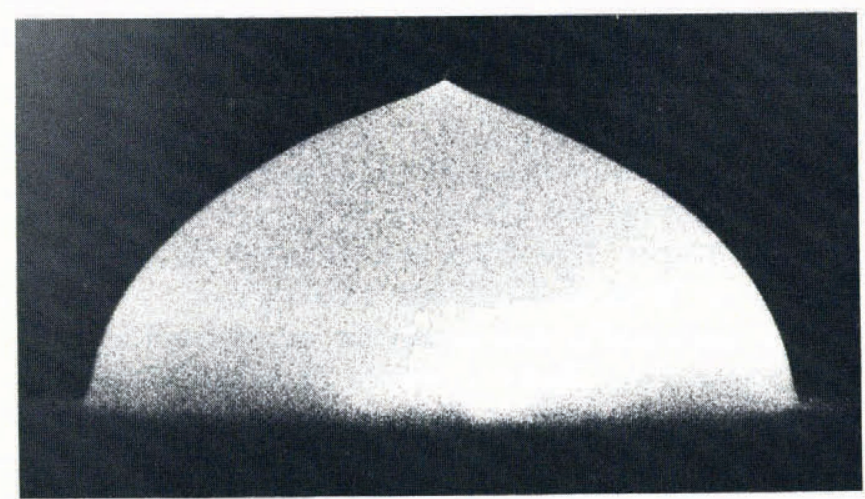

Fig. 2. Photograph of an ice pellet with a well-developed pinnacle. 


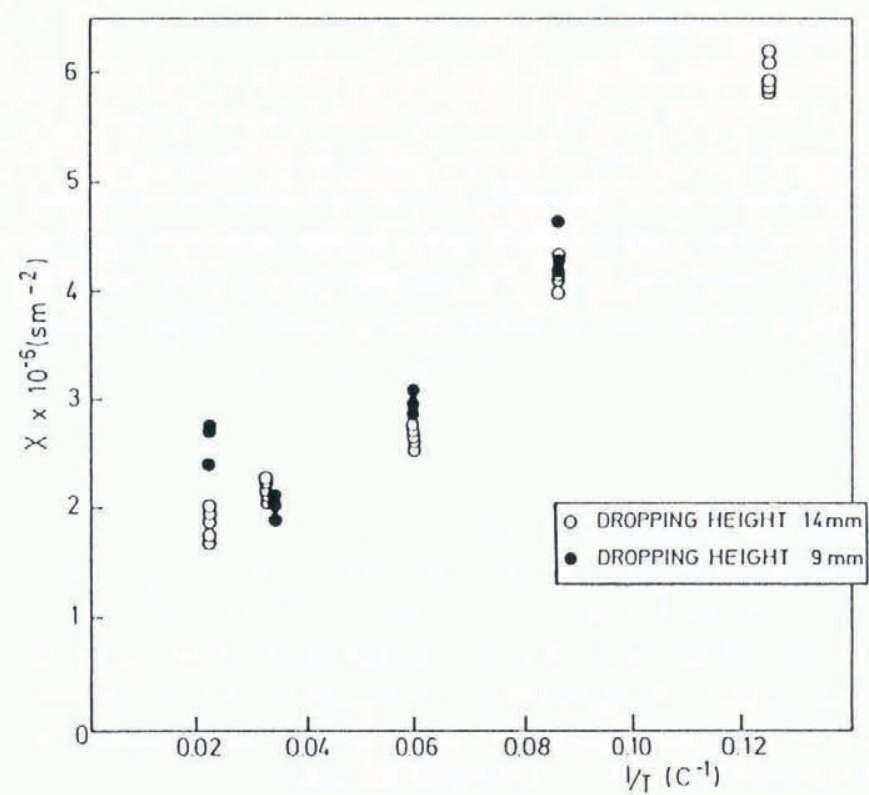

Fig. 3. Variation of $\chi$ as a function of $T^{-1}$ for a dropping height of $8 \mathrm{~mm}$.

the pellet was also measured and the volume, $V$, estimated assuming circular symmetry about the vertical axis. Visual observation justified this assumption, except that in some cases the pinnacle was slightly offset. However, this introduces little error into $V$. The basal diameter, $d$, of the pellet was found by measuring, with a travelling microscope, the diameter of the clearly defined, circular, defrosted area of copper surface revealed when the pellet was removed.

It was found empirically that the quantity $\chi=t d^{2} / V h$ was approximately constant at a fixed temperature. We show in Figure 3 the measured values of $\chi$ plotted against the reciprocal of the temperature depression, $T$, of the metal surface, for two different dropping heights. It can be shown that a straight line through the origin of Figure 3 would, in theory, describe the freezing of a hypothetical, cylindrical water body, by downward conduction of the latent heat of freezing. It can be seen that in fact $\chi$ increases smoothly but non-linearly with $1 / T$. The effects of convection, specific heat, and droplet shape probably account for the observed behaviour. It is only weakly dependent on the impact speed. The freezing process is undoubtedly complicated and we shall not attempt to present a full theory to explain it in detail.

The scatter of the results gives a good indication of the combined effects of experimental error in the measurables $t, d, h$ and $V$, together with any effects due to experimental non-reproducibility.

The critical shear force, $F$, necessary to remove a pellet was measured by applying a gradually increasing, known horizontal force to the ice via a smooth, insulated lever, constrained to swing in the vertical plane through the pellet's position. The arrangement of this is shown in Figure 1. The force was provided, via pulley and thread, by a weighted trolley standing on a smooth inclined slope. To make a measurement of the critical shear force, $F$, we slowly increased the inclination of the slope, by means of a screw. A slowly increasing force was thus transmitted to the ice pellet. The angle of the slope at which the ice became detached was recorded and a value for $F$ calculated knowing the angle, total weight, and the lever geometry. In calibration tests the mechanism worked smoothly, reproducibly and well; frictional effects within the mechanism were very small and an applied force could easily be measured to within a few per cent over the range required. In practice, a difficulty arises concerning the point of contact of the lever with the ice pellet. This is inevitably slightly above the ice-metal interface which means that, as well as the required shear force acting across the ice-metal interface, a small couple also acts. For contact angles in the ice less than $90^{\circ}$, there will be a component of normal force as well. The contact angle and profile of the ice pellet are important in determining the extent of these unwanted effects. In some cases, it was advantageous to adjust the clearance of the lever above the frosted metal surface to a minimum by means of a screw adjuster. Systematic errors arising from these unwanted stresses must be present in our results. The results are, nevertheless, sufficiently reproducible to be of value.

Shear failure always occurred at the ice-metal interface, revealing a near-circular frost-free area, $A$, of bare copper. We measured $d$, the diameter of the contact area and calculated the area $A$. Below $-8^{\circ} \mathrm{C}$, random errors of a few per cent occurred in $A$. Above $-8^{\circ} \mathrm{C}$, the contact area was often not quite circular and the error in $A$ in these cases could be as much as $30 \%$. Figure 4 shows the critical shear stress $\sigma_{0}=F / A$, needed to remove the ice pellets, as a function of the initial temperature of the copper, over the range $-2^{\circ}$ to $-66^{\circ} \mathrm{C}$. Above $-2^{\circ} \mathrm{C}$, no reproducible measurement of $F$ could be made because the ice pellets were too flat. Below $-62^{\circ} \mathrm{C}$, the pellets did not measurably adhere to the surface. Although the experimental points are somewhat scattered, clear trends can be discerned. Between $-2^{\circ}$ and $-22^{\circ} \mathrm{C}, \sigma_{0}$ increases approximately linearly as the temperature falls, the gradient being

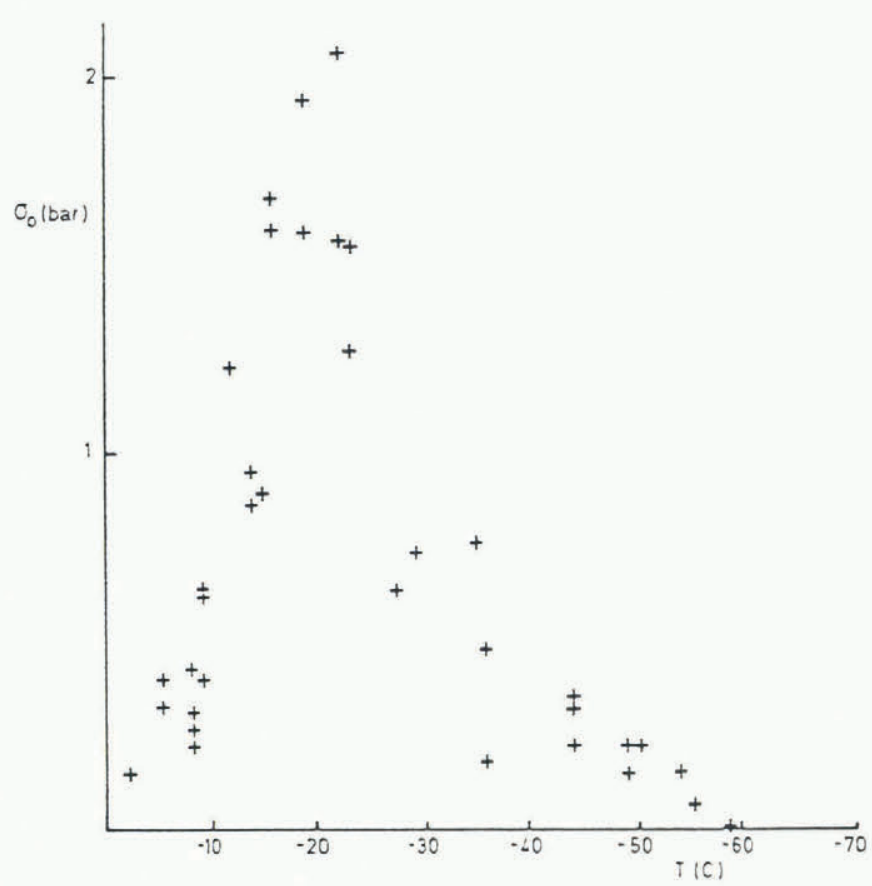

Fig. 4. The critical shear stress $\sigma_{0}$ for detaching an ice pellet from a cold copper surface, as a function of surface temperature, $T$, and dropping height. 
$-1.05 \pm 0.05 \mathrm{bar} \mathrm{K}^{-1}$, reaching a maximum value of $21 \pm$ 1 bar at $-22^{\circ} \mathrm{C}$. Below $-22^{\circ} \mathrm{C}$, it falls monotonically, reaching zero at $-61^{\circ} \pm 3^{\circ} \mathrm{C}$.

The sharp decline in strength below $-22^{\circ} \mathrm{C}$ is perhaps caused by stress cracks initiated at the perimeter of the icecopper interface by differential contraction during freezing. Presumably, the ice-copper junction forms at $0^{\circ} \mathrm{C}$, during the droplet-spreading phase and is subsequently cooled to $T$. Because Young's modulus for copper, $\left(E_{\mathrm{CU}}\right.$ $\simeq 13 \times 10^{5}$ bar), is much greater than that for ice ( $E_{\mathrm{ICE}}$ $\left.\simeq 0.9 \times 10^{5} \mathrm{bar}\right)$, the ice near the interface must conform to the thermal contraction of the copper. For this to occur, a tensile- stress field is needed in the ice, acting parallel to the interface. This is maintained by an interfacial radial shear stress between the copper and the ice. This stress must, by symmetry, be zero at the centre of the contact area. It probably increases smoothly with radius reaching a maximum near the periphery, where adhesion cracks are therefore most likely to form. The mean shear stress will be of order $\left(\alpha_{\mathrm{ICE}}-\alpha_{\mathrm{CU}}\right) \times E_{\mathrm{ICE}} T$. Mean coefficients of linear expansion of ice and copper between $-2^{\circ}$ and $-22^{\circ} \mathrm{C}$ are $\alpha$ $I_{\mathrm{ICE}}=5 \times 10^{-7}$ and $\alpha_{\mathrm{CU}} \simeq 1.7 \times 10^{-7} \mathrm{C}^{-1}$, respectively. Therefore, at $-22^{\circ} \mathrm{C}$ the mean shear stress could reach 63 bar. This stress is larger than the measured strength, but of the same order of magnitude. This must be considered a satisfactory agreement, bearing in mind the order-of-magnitude nature of the stress estimate and the complicated geometry and thermal history of cooling.

\section{CONCLUSION}

Water drops falling gently on to cold copper surfaces freeze to form ice pellets. These adhere most strongly at a temperature of $-22^{\circ} \mathrm{C}$ but are easily detached above $-2{ }^{\circ} \mathrm{C}$ and below $-62^{\circ} \mathrm{C}$. Broadly similar behaviour was observed by Bari and Hallet (1974) when impact velocities of up to $6 \mathrm{~m} \mathrm{~s}^{-1}$ were employed. It remains to be seen whether similar behaviour is found at the much higher impact velocities encountered by high-flying aircraft such as HOTO1.

\section{ACKNOWLEDGEMENT}

The experimental work described in this paper was proposed by Professor M. Lowson and was carried out in the H.H. Wills Physics Laboratory, University of Bristol, by D. Hargreaves and S. Stuart-Smith, as a jointly supervised undergraduate project. We are grateful to Professor J. F. Nye for a helpful discussion.

\section{REFERENCES}

Bari, S. A. and J. Hallett. 1974. Nucleation and growth of bubbles at an ice-water interface. J. Glaciol., 13(69), 489-520

The accuracy of references in the text and in this list is the responsibility of the authors, to whom queries should be addressed. 